\title{
Terror: Einsatzpläne für den Fall, der lieber nicht eintreffen soll
}

Lernen von der Bundeswehr, mehr Austausch und Vernetzung und neue Programme für die rasche Onlinekommunikation - Rettungswesen, Kliniken und Planer üben für den Ernstfall eines Terroranschlags.

Seit Paris ist alles anders, seitdem wird geübt, meint Hans-Georg Jung. Der Leiter der Stabsstelle Medizinische Gefahrenabwehr beim Gesundheitsamt der Stadt Frankfurt am Main sieht allerdings auch keine Möglichkeit mehr, noch irgendwo Zeit zu verschwenden: „Dass jemand nach Paris 2015 immer noch nicht aufgewacht ist, kann ich mir nicht vorstellen.“

Freitagabend, 13. November 2015, 3 Bomben vor dem Stade de France, der Horror im Bataclan, Schüsse aus Kalaschnikows gegen Menschen, die in Straßencafés und Restaurants sitzen - 130 Tote, 352 Verletzte. Das Bekennerschreiben des IS wenige Tage später im Internet spricht von einem „gesegneten Kriegszug“.

Es sind nicht die ersten und nicht die letzten derartigen Anschläge in Europa. Es folgen am 22. März 2016 die Anschläge in Brüssel, 3 Attentäter, die sich am Flughafen und der Metrostation Maalbeek in die Luft sprengen - 32 Tote, mehr als 300 Verletzte. Am 14. Juli 2016 ist es Nizza; 1 Attentäter, der einen LKW in die Passanten steuert, 86 Tote, über 400 Verletzte. Ein sehr ähnliches Muster am 19. Dezember 2016 auf dem Breitscheidplatz in Berlin, 1 Attentäter, ein LKW. Am Ende 12 Tote, über 50 zum Teil schwer Verletzte. Berlin ist der erste Massenanfall von Verletzten (MANV) durch Terror hierzulande.

Immerhin: Das Rettungskonzept habe funktioniert, erklärt der Chef der Berliner Feuerwehr Wilfried Gräfling einen Tag später auf einer Pressekonferenz. 153 Einsatzkräfte waren vor Ort, unterstützt von 80 Helfern, vor allem dem Deutschen Roten Kreuz, haben die Opfer bestmöglich in die umliegenden Kliniken gebracht.

Sind Rettungskräfte und Ärzte also vorbereitet, zumindest die Folgen bestmög- lich zu begrenzen, wenn es zu solchen Anschlägen kommt?

Die Antworten von 2 Experten auf den folgenden Seiten fallen vorsichtig aus und, wie könnte es unter Experten anders sein, ihre Einschätzungen sind im Detail durchaus unterschiedlich.

Für eine Kommunikation zwischen Leitstelle, Rettungskräften, Feuerwehr und Kliniken und schnelle Bergung von Opfern im Ernstfall sei eine Stadt wie Frankfurt heute vorbereitet, betont Hans-Georg Jung (Siehe das Interview ab Seite 381). Fachlich allerdings hätten Notärzte und Rettungskräfte Defizite lange Jahre verschleppt.

Da ist er sich mit dem 2. Interviewpartner der ZfOU zum Thema, Professor Benedikt Friemert vom Bundeswehrkrankenhaus Ulm durchaus einig (Siehe das Interview $a b$ Seite 384). „Gerade zivile Chirurgen hierzulande“, so Friemert, „brauchen neu Kenntnisse in Einsatzchirurgie." Lernen könnten sie es von Ärzten mit Auslandserfahrung bei der Bundeswehr.

Es sind weniger staatliche Stellen, vielmehr vor allem Fachgesellschaften, die derzeit das Thema Vorbereitung und Schulung für mögliche Terroranschläge anschieben. Und dies in neu betonter Zusammenarbeit mit der Bundeswehr.

- Seit Anfang 2013 hat die Deutsche Gesellschaft für Allgemein- und Viszeralchirurgie, DGAV, dafür eine eigene AG, die Chirurgische Arbeitsgemeinschaft Militär- und Notfallmedizin (CAMIN).

- Die Deutsche Gesellschaft für Anästhesie und Intensivmedizin (DGAI) unterhält beim Wissenschaftlichen Arbeitskreis für Notfallmedizin eine Arbeitsgruppe „Taktische Medizin“.

- Die DGU präsentierte im September 2016 zusammen mit der Bundeswehr gleich einen 5-Punkte-Plan zur Vorbereitung angesichts der Risiken eines großen Terroranschlags. Dazu zählen neue Fortbildungsveranstaltungen für Ärzte in Katastrophen-Chirurgie, mehr Zusammenarbeit mit der Gesellschaft für Wehrmedizin DGWMP und eine $\mathrm{Er}$ - weiterung des Traumaregisters um Daten der Opfer von Schuss- und Explosionsverletzungen. Mit der AG Einsatz-, Katastrophen- und Taktische Chirurgie (EKTC) gibt es seit 2013 auch ein institutionalisiertes Forum für den Austausch mit Ärzten der Bundeswehr. Die Vorbereitung für mögliche Katastrophenszenarien habe in der Bundesrepublik lange als Schritt in den Krieg gegolten und sei geächtet gewesen. Jetzt müssten fehlendes Wissen und Techniken neu gelernt werden, so die DGU.

Das Attentat auf dem Breitscheidplatz führte bei aller Perfidie zu Verletzungen, die Medizinern hierzulande prinzipiell noch vertraut sind. Fast noch mehr fürchten Experten auch hierzulande einen Anschlag mit Bomben, Sprengstoffgürtel oder Großkaliberwaffen mitten in Fußgängerzone oder Stadion. Nicht ohne Grund blicken hiesige Chirurgen besonders regelmäßig nach Israel. Das Land hat unter allen westlichen Ländern die meisten, alias schlimmsten Erfahrungen mit diesem Terror.

- Zu rechnen ist nach Anschlägen mit besonders vielen jugendlichen Opfern, erklärte der israelische Traumaexperte Kobi Peleg auf dem letzten DKOU in Berlin. Denn die Attentäter suchten die „Crowdedness“, Busse, Stadien oder öffentliche Plätze, die wiederum vor allem junge Menschen als Bühne nehmen.

- Die Opfer leiden vor allem nach Bombenanschlägen an multiplen Verletzungen und verschiedenen Verletzungsarten. Besonders hohen Tribut fordern Nägel oder Schrauben, die Attentäter zusätzlich in ihre Bomben packen, und die als Geschosse verheerend wirken. Über die Hälfte der Opfer solcher Attentate erleidet nach Zahlen aus Israel und Madrid dadurch schwere Verletzungen am Kopf.

- Kliniken müssen sich auf einen hohen Bedarf an Bluttransfusionen einstellen, wie Gidon Almogy vom Hassadah Medical Center in Jerusalem auf dem letzten DKOU berichtete. Jedes 3. Opfer nach Bombenanschlägen in den versorgen- 
den Kliniken in Israel braucht Transfusionen - wie eine Auswertung von Daten aus Traumazentren in Jerusalem zwischen 2001 und 2005 zeigt.

- Wichtig ist eine gute Abstimmung und Logistik aller Einsatzkräfte, um nach Möglichkeit zu verhindern, dass nächstgelegene Krankenhäuser die ganze Verletztenlast schultern müssen. Mittelbis Schwerverletzte sollten möglichst gut auf Kliniken verteilt werden, um einen Kollaps einzelner Häuser zu vermeiden, betonte Kobi Peleg in Berlin. Auch in Israel habe man das erst lernen müssen. Und besonders gut kümmern müsse man sich, so paradox es auf den ersten Blick wirkt, auch und gerade um die Leichtverletzten Die dürfen im Notfall nicht als Selbsteinweiser in großer Zahl in den Kliniken auftauchen, drohten dann alles zu blockieren. Ein Thema, das mittlerweile auch hiesige Einsatzpläne auf dem Schirm haben (Siehe das Interview Jung).

Fakt ist, Deutschland kann nicht eins zu eins Erfahrungen aus anderen Ländern für das eigene Rettungswesen übernehmen. Israelische Experten verweisen vor allem auf die zentrale Organisation des gesamten Rettungswesens in ihrem Land als Schlüssel zu möglichst schneller und guter Versorgung nach Attentaten. Das gesamte Rettungswesen wird in Israel vom Gesundheitsministerium geleitet. Regelmäßige Teilnahme an Übungen in Notfallrettung ist für Kliniken und Ärzte landesweit Pflicht. Eine gemeinsame Terminologie landesweit, enge Zusammenarbeit ziviler und militärischer Stellen, bei Bedarf zentrale Koordination aller Akteure durch die Polizei, beschreiben Kobi Peleg und Bruria Adini in einem Übersichtsartikel 2013 als Elemente einer guten koordinierten Versorgung nach Terrorattacken. Ergänzt wird dies durch ein landesweites Onlinesystem, das allen Akteuren die Neuaufnahmen und die noch freien Kapazitäten der Kliniken anzeigt.

Deutschland hat exakt entgegengesetzte Strukturen. Rettung nach Terroranschlägen ist hierzulande Ländersache. Etliche Bundesländer überarbeiten derzeit ihre Richtlinien. Bayern etwa hat Ende 2016 seine Richtlinie zur Bewältigung von Ereignissen mit einem Massenanfall von Notfall- patienten und Betroffenen novelliert auch in Hinsicht auf Terrorgefahren.

Das Bundesamt für Bevölkerungsschutz und Katastrophenmedizin (BBK) greift wenn, dann erst dann ein, nachdem großflächig Infrastruktur zerstört wäre. Ansonsten betont die Einrichtung des Bundesinnenministeriums, bei einem Terroranschlag nicht zuständig zu sein: „Das BBK übernimmt aufgrund der verfassungsrechtlichen Aufgabenteilung keine aktive Rolle bei der Rettung und medizinischen Versorgung der Opfer“, so das BBK auf Anfrage der ZfOU. Allerdings könnte das BBK von einem Bundesland um Amtshilfe, beziehungsweise um Katastrophenhilfe gebeten werde und bestimmte Koordinierungsaufgaben übernehmen, etwa die „Abfrage von freien Spezial-Krankenhausbetten in Nachbarländern Deutschlands“.

Für extreme Katastrophenfälle, sogenannte Ereignisse der Versorgungsstufen 3 und 4 mit zerstörter Infrastruktur, alias Zusammenbruch von Internet, Telefonie, Funk, Strom und Wasser, baut das BBK 61 sogenannte Medizinische Task Forces (MTF) auf, die dann auch überregional helfen sollen. Auch sie aber, so die Behörde, stünden wenn, dann „unter der Führung/Leitung der jeweils betroffenen lokalen/regionalen Einsatzleitung“.

Klar ist: Die starke Aufgliederung des Rettungswesens auf Länder und dort meist delegiert an Leitstellen jeweils pro Landkreis und/oder Kommune schafft hierzulande einige Schnittstellen mehr für eine gute Abstimmung und Koordination. Denn im Notfall müssten Patienten auch überregional rasch verteilt werden. Vielerorts, selbst in Großstädten, setzen Rettungskräfte und Mediziner noch auf Telefon und Fax. In Köln alarmiert die Feuerwehr bei einem Großschadensereignis zunächst per Fax 17 Notfallkrankenhäuser, was dort wiederum die jeweiligen Notfallpläne in Gang setzt.

Mancherorts entstehen Bottom-up-Lösungen für überregionale und vor allem rasche Vernetzungen. In Hessen setzt man heute auf eine Software zur Onlinekommunikation zwischen Rettungsdiensten, Leitstellen und Kliniken. Das System IVENA erlaubt eine Allokation der Patienten an- hand einer vor Ort vom Rettungsdienst geleisteten Erstdiagnose in jeweils spezialisierte Krankenhäuser (Siehe das Interview Jung). Vor allem aber erlaubt es im Krisenfall auch eine Abstimmung über kommunale Grenzen hinweg.

Nicht nur beim Thema Schulung von Rettungskräften und Ärzten übernehmen derweil vor allem einzelne Fachgesellschaften die Initiative. Das Traumanetzwerk der DGU mit seinen rund 600 spezialisierten und zertifizierten Kliniken bietet obendrein eine Struktur zur Versorgung von Verletzten über Regionen oder Bundesländer hinweg.

Aber längst nicht überall sieht man sich wirklich gut aufgestellt. Noch im März dieses Jahres zitiert eine Panorama-Sendung des NDR den Deutschen Berufsverband Rettungsdienst, der das Fehlen einheitlicher Rettungskonzepte in den norddeutschen Bundesländern moniert. Die Antworten mancher der von Panorama befragten Behörden und Innenministerien von Ländern lesen sich wie reine Absichtserklärungen - ein landesweites Konzept zur Zusammenarbeit aller Dienste und Krankenhäuser, das für Schleswig-Holstein gerade die Polizei entwickelt, steckte da noch in der Abstimmung, es soll, wenn fertig, auch „beschult“ und „geübt“ werden. Etliche Behörden wussten zum Beispiel auch nicht, inwieweit Tourniquets in den Rettungswagen in ihrem Bundesland vorrätig waren.

Noch Anfang 2017 forderten DGU wie DGAI erneut, dass alle Rettungsfahrzeuge bundesweit diese Notfallinstrumente erhalten. Abbindesysteme, bekannt aus der „Einsatzmedizin“, mit denen sich gefährliche Blutungen stoppen und Leben retten lassen. In Bayern, loben die Fachgesellschaften, müssen Rettungswägen heute Tourniquets und weitere Materialien für den Notfall bereithalten, andere Bundesländer hinkten hinterher.

Aber die Handhabung auch dieser Instrumente will gelernt sein. Wenn, dann müssen Tourniquets schon richtig festgezogen werden, bis zum „Pulsverlust“, wie die DGAl in einer neuen „Handlungsempfehlung“ erklärt. Und andererseits gibt es auch Risiken auf Komplikationen - zu lan- 
ges Abbinden kann zu Nervenläsionen oder einem Kompartmentsyndrom führen. So lange ein Druckverband reicht, ist damit erst mal der angesagt.
Üben, bitte! Auch wenn im Zweifel etwas Glück hinzukommen muss: Wer gut vorbereitet sei, habe zumindest eine 50:50Chance, die Versorgung nach einem großen Anschlag ordentlich über die Bühne zu kriegen, weiß Hans-Georg Jung. „Ora- keln kann ich nicht, keiner weiß, was kommt" schränkt der Experte ein. Dass allen lieber wäre, es kommt gar nichts - keine Frage. Hilft nur nicht.

Bernhard Epping 